\title{
MMI filters configuration for dual- wavelength generation in a ring cavity erbium-doped fibre laser
}

\author{
Ricardo I. Álvarez-Tamayo1, José G. Aguilar-Soto1', Manuel Durán-Sánchez ${ }^{1,2}$, José E. Antonio-López, \\ Baldemar Ibarra-Escamilla ${ }^{* *}$ and Evgeny A. Kuzin ${ }^{1}$
}

\begin{abstract}
Background: Dual wavelength laser generation has been of constant interest due to their applications in optical communications and teraheartz generation. A novel configuration for Dual-wavelength laser generation based on the use of a couple of multimode interference (MMI) filters is demonstrated in a ring cavity Erbium-doped fibre laser.

Methods: The MMI filters consist of a segment of no-core fibre spliced between two SMF-28 single mode fibre segments. The MMI filters configured as a Mach-Zehnder interferometer, are used as transmission spectral filters for simultaneous generation of two laser wavelengths. An optical attenuator is used to adjust the intra-cavity losses for dual-wavelength laser generation.
\end{abstract}

Results: Laser emission at 1540.4 and $1554 \mathrm{~nm}$ for a wavelength separation of $~ 13.6 \mathrm{~nm}$ is obtained. The laser wavelengths output power stability variations with the applied pump power is also experimentally discussed.

Conclusions: The use of MMI filters in the proposed dual-wavelength laser filter is experimentally demonstrated as a reliable device for dual-wavelength generation in fibre lasers.

Keywords: Fibre lasers, Multimode interference filter, Dual-wavelength laser, Erbium-doped fibre

\section{Background}

Spectral filters in optical fibre based on single-modemultimode-single-mode (SMS) fibre structure have been of significant interest as reliable optical devices because of their many advantages such as compatibility for all-fibre integration, low cost, low insertion loss and ease to fabricate. The operation principle of the SMS fibre structure is based on a self-imaging phenomenon described for slab waveguides by Soldano and Penings [1]. The filtering aspects of the SMS fibre structure was discussed by Mohammed et al. [2]. The SMS filter exhibits a narrow transmission spectral width with a leading wavelength peak. Moreover, its transmission wavelength peak can be tuned by external parameters such as temperature, strain and liquid refractive index $[3,4]$. This features make the SMS fibre structure

\footnotetext{
* Correspondence: baldemar@inaoep.mx

${ }^{1}$ Optics Department, Instituto Nacional de Astrofísica, Óptica y Electrónica, Luis Enrique Erro 1, Puebla 72824, Mexico

Full list of author information is available at the end of the article
}

attractive for fibre-optical sensing [5-7] and tuneable fibre laser applications [8-10]. The performance of the SMS structure as a band-pass filter $[8,9]$ make it a reliable device for wavelength selection and tuning of ring cavity fibre lasers. Furthermore, when the MMI filter is properly designed, the transmission peak wavelength can be easily selected in terms of the multimodal fibre (MMF) refractive index, core diameter and length $[2,3]$.

As the dual-wavelength laser emission has generated a considerable interest in recent years, the obtaining of two simultaneous laser wavelengths by using a single cavity has been attractive in different areas such as optical fibre sensing, optical communications, microwave and terahertz generation. However, EDF is a homogeneous gain medium at room temperature, which leads to a strong mode competition for the generated laser lines. Therefore, several techniques to achieve dual-wavelength laser emission where the use of optical filters as a reliable method for cavity losses adjustment have been reported [11-15], among which can 
be mentioned fibre Bragg gratings (FBG), Mach-Zehnder interferometer (MZ) and Sagnac interferometer. Recently, tuneable Erbium- and Ytterbium-doped fibre lasers using MMI filters have been reported [8-10]. However, the potential of using MMI filters for dual-wavelength laser generation, has been underexploited. To our knowledge, only few researches of dual-wavelength fibre lasers in which a SMS structure is used for dual-wavelength generation have been reported [16-18]. However, the use of a single SMS structure in these investigations, limits the laser lines wavelength separation and make difficult the wavelength selection and the cavity losses adjustment.

In this paper, we demonstrate stable dual-wavelength laser emission of an EDF laser based on the use of two SMS filters. The filters with SMS structure are disposed in a MZ interferometer configuration for separately laser wavelength selection. The dual-wavelength laser lines separation is $\sim 13.6 \mathrm{~nm}$ with laser lines wavelength at 1540.4 and $1554 \mathrm{~nm}$. The wavelength is marginally modified with the increase of the pump power. The reliability of using two MMI filters for separately wavelength selection of stable dual-wavelength laser operation is experimentally demonstrated.

\section{Methods}

MMI dual-wavelength filter principle and characterization Figure 1a shows the schematic of the SMS structure used in our experiments. A segment of multimode nocore fibre (NCF) is spliced between two segments of standard single mode fibre (SMF-28).

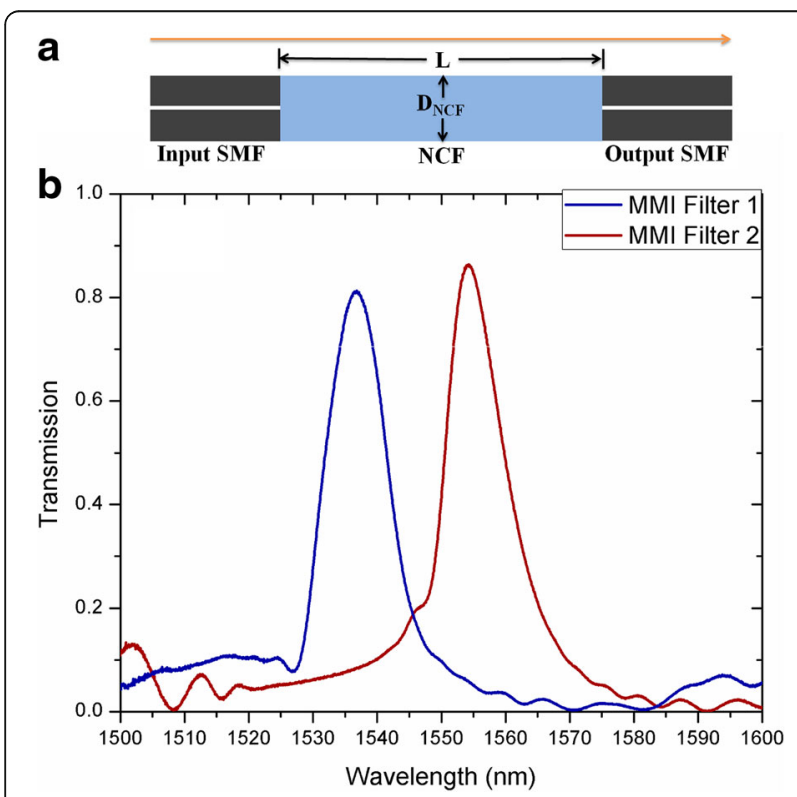

Fig. 1 a Schematic of the proposed MMl filters structure, $\mathbf{b}$ Transmission spectral response of the MMl filter 1 (blue line) and MMI filter 2 (red line)
The SMS structure has a narrow band-pass response with a wavelength peak based on the multimodeinterference self-imaging effect. The modes are excited in the NCF when a field is launched from the input SMF. The interference of these excited modes produces selfimages at periodic intervals along the NCF. The central wavelength of the SMS filter can be expressed as [4]:

$$
\lambda_{0}=p \frac{n_{N C F} D_{N C F}^{2}}{L},
$$

where $p$ is the self-image number, $n_{N C F}$ is the NCF effective refractive index, $D_{N C F}$ is the diameter of the NCF corresponding to the effective width of the fundamental mode, and $L$ is the NCF length. The homemade MMI filters were constructed to obtain the fourth self-image by using a NCF (in which air acts as cladding) with $125 \mu \mathrm{m}$ diameter. The NCF lengths of 58.7 and $58 \mathrm{~mm}$, to obtain transmission wavelength peaks of 1537.5 and $1554.3 \mathrm{~nm}$ (for MMI filter 1 and MMI filter 2 respectively) were calculated with Eq. (1). Fig. 1b shows the transmission of the constructed MMI filters. To measure the transmission, we used a LED source with emission in a wavelength range from 1465 to $1650 \mathrm{~nm}$, as input signal. The transmission of the MMI filters was estimated by the following method: Initially, three spliced segments of SMF-28 fibre were mounted in a metal plate to avoid instability in the estimation, and the fibre ends were connected to bare fibre adaptors. In one of the adaptors the LED source was connected and the input signal spectrum was recorded with an OSA at the other connector. Then, the central segment of SMF-28 fibre was replaced with one of the MMI filters. Likewise, the output signal of the LED source due to the MMI was measured. The transmission of both separately MMI shown in Fig. 1b was estimated as the measured MMI output signal divided by the measured LED source output signal. As it can be observed, the transmission spectrum of the MMI filter 1 (blue line) exhibits a wavelength peak at $\sim 1538.6 \mathrm{~nm}$ and for the MMI filter 2 (red line) a wavelength peak is observed at $\sim 1554.3 \mathrm{~nm}$. The FWHM of both MMI filters is around $11 \mathrm{~nm}$. The transmission losses for the MMI filter 1 and MMI filter 2 are $19 \%$ and $\sim 14 \%$, respectively.

Figure 2 shows the configuration and the spectral response of the proposed dual-wavelength filter with two MMI filters (MMI-DWF) used to obtain dual wavelength laser emission in a ring cavity fibre laser. To achieve both transmissions with independent performance, the MMI filters were disposed between two 50/50 fibre couplers (coupler 1 and coupler 2) in a MZ interferometer configuration as it is shown in Fig. 2a. The filter is mounted on a metal plate to avoid instability of the laser performance due to mechanical deformation. 


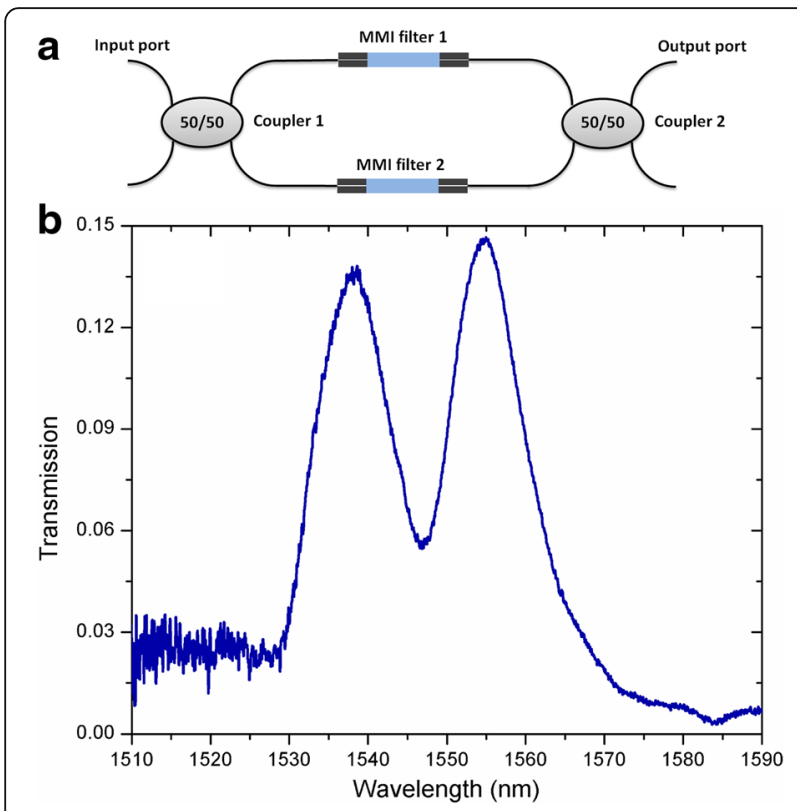

Fig. 2 a Schematic of the proposed MMI-DWF, b MMI-DWF transmission spectral response

The interference modulation is avoided due to the long path difference between the two arms of the MZ interferometer. Figure $2 \mathrm{~b}$ shows the transmission of the MMI-DWF. The transmission spectrum exhibits two wavelength peaks at 1538.5 and $1554.6 \mathrm{~nm}$ corresponding to the MMI filter 1 and the MMI filter 2, respectively. As it can be observed, the longer wavelength peak presents slightly higher transmission than the shorter wavelength peak, in accordance with the individual MMI filters transmission spectra shown in Fig. 1b.

\section{Results and discussions}

The experimental setup for the dual-wavelength EDF laser using the proposed MMI-DWF is shown in Fig. 3. A 3-m length EDF (MetroGain M-12 980/125) with

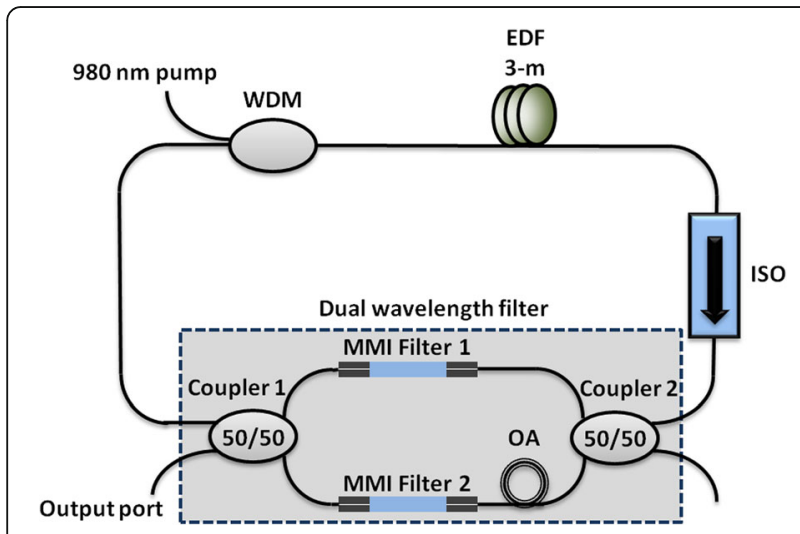

Fig. 3 Experimental setup of the ring cavity dual-wavelength EDF fibre laser with the MMI-DWF absorption of $20 \mathrm{~dB} / \mathrm{m}$ at $1531 \mathrm{~nm}$ and numerical aperture (NA) of 0.24 was used as a gain medium. The EDF is pumped by a $980 \mathrm{~nm}$ single mode laser diode with maximal output power of $120 \mathrm{~mW}$ through a 980/ $1550 \mathrm{~nm}$ wavelength-division multiplexer (WDM). A polarization independent optical isolator (ISO) is placed to force unidirectional ring laser operation. As it was shown in Fig. 2b, the longer wavelength peak of the MMI-DWF exhibits a higher transmission than the shorter wavelength. For intra-cavity losses adjustment to achieve stable dual-wavelength laser operation, an optical attenuator (OA) was inserted in the MMI-DWF arm containing the MMI filter 2 to add losses by curvature on the MMI filter with higher transmission. The coupler 1 output port taken as the laser output is used to measure and analyze the laser spectrum by an optical spectrum analyzer (OSA, Yokogawa AQ6375) with scanning range from 1200 to $2400 \mathrm{~nm}$ and spectral resolution of $0.05 \mathrm{~nm}$.

Figure 4 shows the EDF laser output spectra at pump power of $100 \mathrm{~mW}$. Figure 4a shows the simultaneous dual-wavelength laser emission with equal output powers at laser wavelengths $\lambda_{1}=1540.4 \mathrm{~nm}$ and $\lambda_{2}=$ $1554 \mathrm{~nm}$, where $\lambda_{1}$ and $\lambda_{2}$ are the generated laser wavelengths due to the MMI filter 1 and the MMI filter 2, respectively. The cavity losses adjustment performed by the OA requires similar amplification gain at the generated wavelengths. The MMI filters wavelengths were chosen where the EDF amplification spectrum profile exhibits a flat zone proper for cavity losses balancing to reach dual wavelength laser generation.

Figure $4 \mathrm{~b}$ shows repeated measurements of the dualwavelength laser output with a launched pump power of $100 \mathrm{~mW}$. The OA was adjusted to obtain dual wavelength laser operation with equal power wavelengths. Once simultaneous laser emission was reached, the OA was fixed to prevent instability by deformation. A set of ten measurements with a 2 min interval were obtained at room temperature in which thermal dependence of the MMIDWF is not noticeable. The peak power variation for each generated wavelength was less than $0.3 \%$. Therefore, dual-wavelength laser emission stability is observed.

Figure 5 shows the output power of the generated laser lines as a function of the pump power. The measurements were obtained for pump powers from 40 to $120 \mathrm{~mW}$ with an interval of $20 \mathrm{~mW}$. A set of 10 measurements with interval of 2 min was performed at each pump power. With a pump power of $80 \mathrm{~mW}$, dualwavelength laser emission with equal powers was initially set by adjusting the OA. For the subsequent measurements with different pump powers, the same OA adjustment was used. As it can be observed at pump power of 40 and $60 \mathrm{~mW}$, the output power of $\lambda_{1}$ is higher than for $\lambda_{2}$ and the output power of both laser 

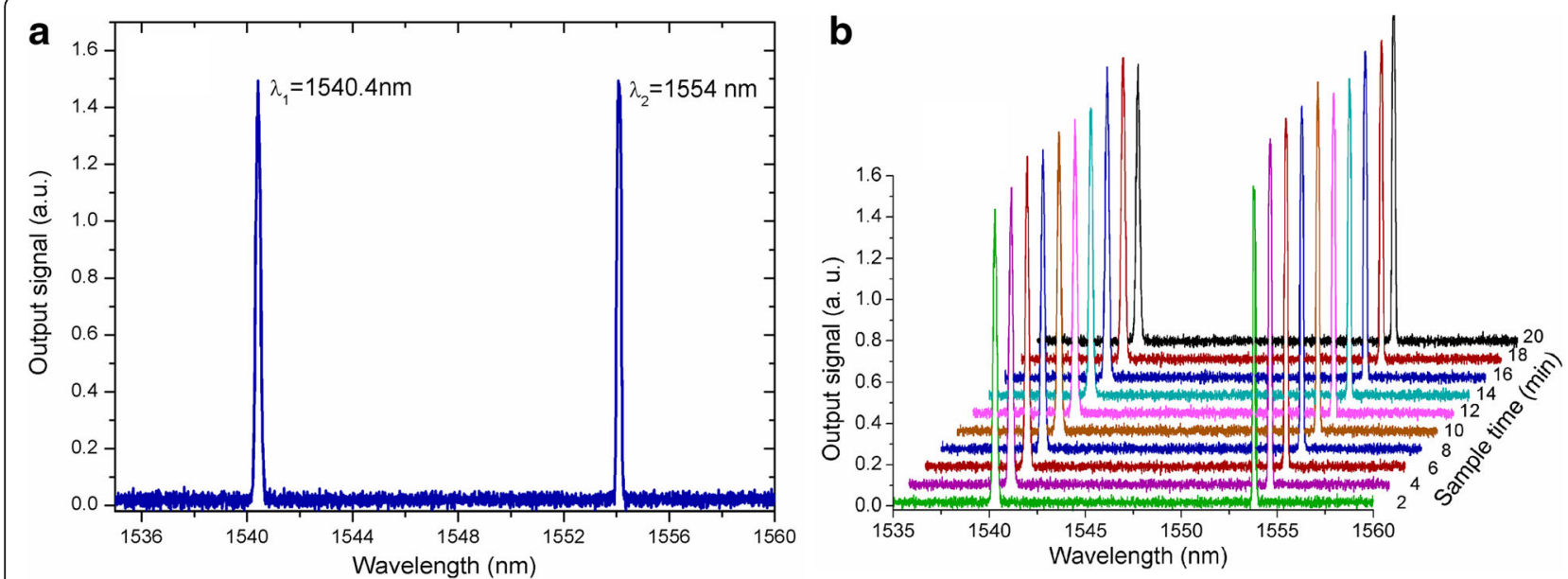

Fig. 4 Output spectra of the dual-wavelength laser. a laser line are $\lambda_{1}=1540.4 \mathrm{~nm}$ and $\lambda_{2}=1554 \mathrm{~nm}$, $\mathbf{b}$ stability of dual-wavelength fibre laser

lines remains stable. At pump power of 80 and $100 \mathrm{~mW}$ stable dual-wavelength laser operation with equal power is observed. At pump power of $120 \mathrm{~mW}$, the power became unstable.

Figure 6 shows the laser wavelengths behaviour as a function of the pump power. The measurements were performed with the same settings used to obtain the measurements shown in Fig. 5. With a pump power of $80 \mathrm{~mW}$ an initial adjustment in the OA was performed to achieve dual-wavelength laser operation with equal power. As is shown in Fig. 6a, laser lines displacement toward shorter wavelengths is observed with increment of the pump power. The wavelength displacement for the longer wavelength is larger than for the shorter wavelength, resulting in a variation of the wavelength separation between generated laser lines. With a pump power of $40 \mathrm{~mW}$ the separation between the laser wavelengths is $\sim 13.4 \mathrm{~nm}$ whereas a

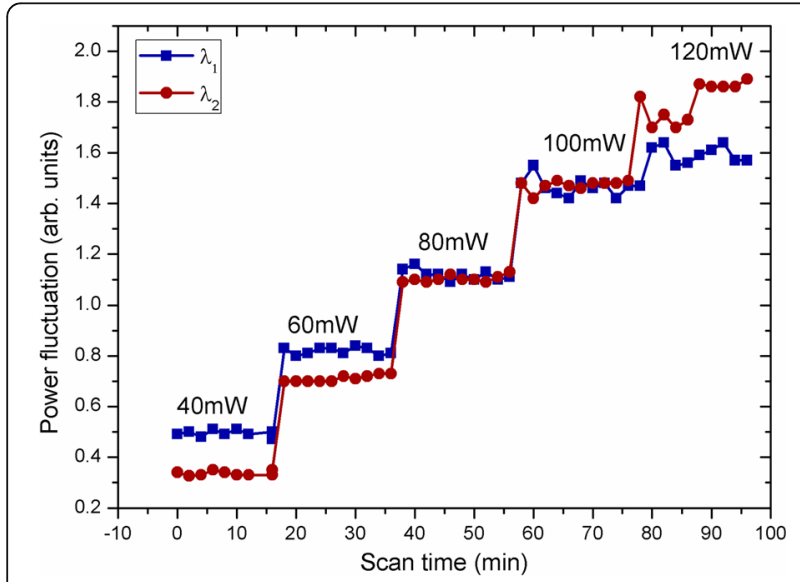

Fig. 5 Dual-wavelength laser lines output power as a function of the pump power separation of $\sim 14.2 \mathrm{~nm}$ is observed with a pump power of $120 \mathrm{~mW}$. Figure $6 \mathrm{~b}$ shows the wavelength displacement $\Delta \lambda$ as a function of the pump power where $\lambda_{1}$ is the shorter wavelength and $\lambda_{2}$ the longer wavelength. $\Delta \lambda$ is the wavelength shift from the initial generated peak wavelengths with a pump power of $40 \mathrm{~mW}$. The wavelength displacement for $\lambda_{2}$ is larger than the observed for $\lambda_{1}$, where the maximal wavelength displacements for $\lambda_{1}$ and $\lambda_{2}$ are -0.7 and $-1.42 \mathrm{~nm}$, respectively.

The laser generation depends on the EDF amplification spectrum gain and on the filter transmission at the specific wavelength. The laser wavelength which will be generated is defined by the maxima of the product of the filter transmission and the EDF amplification. However, each MMI filter exhibits a narrow wavelength range of $2 \mathrm{~nm}$ were the transmission maxima is approximately the same. Therefore, when the pump power is changed, the EDF amplification spectrum undergoes a profile modification that leads to a laser line generation in a slightly shifted wavelength. We attributed the larger shift of the longer wavelength to the fact of $\lambda_{2}$ is generated in a wavelength range in which the EDF amplification spectrum exhibits a downward slope of gain which leads to a more pronounced wavelength shift when pump power is varied.

\section{Conclusions}

In this paper, stable dual-wavelength laser emission using two MMI filters (configured as a MZ interferometer) was experimentally demonstrated. The novel filter structure was used for individual wavelengths selection and cavity losses adjustment to achieve dualwavelength laser operation of an EDF ring cavity laser. With a pump power of $100 \mathrm{~mW}$, dual-wavelength laser emission at 1540.4 and $1554 \mathrm{~nm}$ with a wavelengths separation of $13.6 \mathrm{~nm}$ was achieved. The laser wavelengths output power stability variations with the 



Fig. 6 Dual-wavelength laser emission as a function of the pump power, a Output laser spectrum for different pump powers, $\mathbf{b}$ Laser lines wavelength displacement versus pump power

applied pump power was also experimentally discussed. The wavelength of the generated laser lines slightly shifts toward shorter wavelengths with an increase of the applied pump power. The obtained results demonstrate the reliability of the proposed MMI-DWF for dual-wavelength laser generation in an all-fibre ring cavity fibre laser with potential applications in optical communications and sensing.

\section{Acknowledgements}

Manuel Durán-Sánchez was supported by Cátedras-CONACyT project 2728. R. I. Álvarez-Tamayo wants to thanks CONACyT postdoctoral fellow 160248. B. Ibarra-Escamilla was supported by CONACyT Grants No. 237855 and No. 255284.

\section{Authors' contributions}

RIAT and MDS conceived the work. MDS did the implementation, obtained the experimental results and contributed to results analysis. RIAT did results analysis and contributed to writing. JGAS did the MMI filters design and analysis. JEAL developed and fabricated the no-core fibre and contributed to writing. BIA and EAK coordinated the work, did result analysis and contributed to writing. All authors read and approved the final manuscript.

\section{Competing interests}

The authors declare that they have no competing interests.

\section{Author details}

Optics Department, Instituto Nacional de Astrofísica, Óptica y Electrónica, Luis Enrique Erro 1, Puebla 72824, Mexico. ${ }^{2}$ CONACyT Research Fellow Instituto Nacional de Astrofísica, Óptica y Electrónica, Luis Enrique Erro 1, Puebla 72824, Mexico. ${ }^{3} \mathrm{CREOL}$, The College of Optics and Photonics, University of Central Florida, Orlando, FL 32816-2700, USA.

Received: 19 July 2016 Accepted: 27 October 2016

Published online: 04 November 2016

\section{References}

1. Soldano, LB, Pennings, ECM: Optical multi-mode interference devices based on self imaging: Principles and applications. J Lightw Technol. 13, 615-627 (1995)

2. Mohammed, WS, Smith, PWE, Gu, X: All-fibre multimode interference bandpass filter. Opt. Lett. 31, 2547-2549 (2006)

3. Wang, P, Brambilla, G, Ding, M, Semenova, Y, Wu, Q, Farrell, G: Investigation of single-mode-multimode-single-mode and single-mode-taperedmultimode-single-mode fiber structures and their application for refractive index sensing. J. Opt. Soc. Am. B 28, 1180-1186 (2011)
4. Antonio-Lopez, JE, Castillo-Guzman, A, May-Arrioja, DA, Selvas-Aguilar, R, LiKamWa, P: Tunable multimode-interference bandpass fiber filter. Opt. Lett. 35, 324-326 (2010)

5. Taue, S, Matsumoto, Y, Fukano, H, Tsuruta, K: Experimental analysis of optical fiber multimode interference structure and its application to refractive index measurement. Japanese J Appl Phys 51, 04DG14 (2012)

6. André, RM, Biazoli, CR, Silva, SO, Marques, MB, Cordeiro, CMB, Frazão, O: Multimode interference in tapered single mode-multimode-single mode fiber structures for strain sensing applications. Proceedings of SPIE, OFS2012 22nd International Conference on Optical Fibre Sensors, 84213B, (2012)

7. Antonio-Lopez, JE, Castillo-Guzman, A, May-Arrioja, DA, Selvas-Aguilar, R, LiKamWa, P: Fiber-optic sensor for liquid level measurement. Opt. Lett. 36, 3425-3427 (2011)

8. Castillo-Guzman, A, Antonio-Lopez, JE, Selvas-Aguilar, R, May-Arrioja, DA, Estudillo-Ayala, J, LiKamWa, P: Widely tunable erbium-doped fiber laser based on multimode interference effect. Opt. Express 18, 591-597 (2010)

9. Mukhopadhyay, PK, Gupta, PK, Singh, A, Sharma, SK, Bindra, KS, Oak, SM: Note: Broadly tunable all-fiber ytterbium laser with $0.05 \mathrm{~nm}$ spectral width based on multimode interference filter. Rev Sci Instr 85, 056101 (2014)

10. Selvas, R, Torres-Gomez, I, Martinez-Rios, A, Alvarez-Chavez, JA: Wavelength tuning of fiber lasers using multimode interference effects. Opt. Express 13, 9439-9445 (2005)

11. Liu, Z, Liu, Y, Du, J, Yuan, S, Dong, X: Switchable triple-wavelength erbiumdoped fiber laser using a single fiber Bragg grating in polarizationmaintaining fiber. Opt Comm. 279, 168-172 (2007)

12. Han, $Y$, Lee, JH: Switchable dual wavelength erbium-doped fiber laser at room temperature. Microwave Opt Technol Lett. 49, 1433-1435 (2007)

13. Meng, Y, Zhang, S, Wang, X, Du, J, Li, H, Hao, Y, Li, X: Tunable double-clad ytterbium-doped fiber laser based on a double-pass Mach-Zehnder interferometer. Opt Laser Eng. 50, 303-307 (2012)

14. Álvarez-Tamayo, R.I, Durán-Sánchez, M, Pottiez, O, Kuzin, EA, Ibarra-Escamilla, B, Flores-Rosas, A: Theoretical and experimental analysis of tunable Sagnac highbirefringence loop filter for dual-wavelength laser application. Appl Optics. 50, 253-260 (2011)

15. Durán-Sánchez, M, Álvarez-Tamayo, RI, Pottiez, O, Ibarra-Escamilla, B, Hernández-García, JC, Beltrán-Pérez, G, Kuzin, EA: Actively Q-switched dualwavelength laser with double-cladding $\mathrm{Er} / \mathrm{Yb}$-doped fiber using a $\mathrm{Hi}-\mathrm{Bi}$ Sagnac interferometer. Laser Phys. Lett. 12, 025102 (2015)

16. Ma, L, Kang, Z, Qi, Y, Jian, S: Tunable dual-wavelength fiber laser based on an MMI filter in a cascaded Sagnac loop interferometer. Laser Phys. 24, 045102 (2014)

17. Antonio-Lopez, JE, Sanchez-Mondragon, JJ, LiKamWa, P, May-Arrioja, DA: Tunable Dual-Wavelength Erbium-Doped Fibre Ring Laser. Frontiers in Optics 2012/Laser Science XXVIII, OSA Technical Digest (online) (Optical Society of America, 2012), FW3A.32 (2012)

18. Zhang, P, Wang, T, Ma, W, Dong, K, Jiang, H: Tunable multiwavelength Tmdoped fibre laser based on the multimode interference effect. Appl Optics. 54, 4667-4671 (2015) 